BENM 2021

International Scientific and Practical Conference "Biotechnology, Ecology, Nature Management"

\title{
RHEOLOGICAL PROPERTIES OF SUSPENSIONS
}

\author{
A. T. Vasyukova (a)*, I. U. Kusova (a), A. E. Alekseev (b) \\ *Corresponding author \\ (a) Moscow State University of Food Production, 11, Volokolamskoe highway, Moscow, 125080, Russia, \\ vasyukova-at@yandex.ru \\ (b) K.G. Razumovsky Moscow State University of Technologies and Management (the First Cossack University), 73, \\ Zemlyanoy Val str., Moscow, Russia
}

\begin{abstract}
The article presents data on the effect of additives of pre-processed powder from vegetable raw materials (tomato, parsley, dill, apple, carrot, soy and Jerusalem artichoke) on the structure of fresh batter prepared in various liquid media (water, milk). Various amounts of a modifier based on the use of rice flour made from whole grains, a dispersed powder of herbal dietary supplements were added to the flour suspension. Studies have show that the addition of a small amount of plant powder $(1.0,3.0 \%)$ allows you to modify the microstructure of the resulting suspensions. This modification includes the refining of rice starch grains, stabilization of the non-splitting structure with reduced sizes of matrix inclusions of solid particles of plant biologically active additives, as well as the formation of spherical inclusions of rice starch pasteurized at 50 and $100 \mathrm{oC}$. At the same time, the addition of modifiers such as herbal additives has little effect on the morphology of a batter consisting of a conditioned aqueous medium and fillers.
\end{abstract}

2672-8575 (C) 2022 Published by European Publisher.

Keywords: Dietary supplements, food, suspension, vegetable powder 


\section{Introduction}

The nature of the behavior of various food materials during processing in the working zones of machines and apparatus is determined by the peculiarities of their structural structure. Many food products are inhomogeneous dispersed systems, consisting of two parts: a continuous, that is, a dispersion medium, and a dispersed phase entering it as a content. Depending on the degree of dispersion, the systems are divided into coarsely dispersed (particles of the dispersed phase less than 10-3 cm), microheterogeneous (10-3 ... 10-5 cm) and ultramicroheterogeneous or colloidal (10-5 .. 10-7 cm). As the particle size decreases, the surface area increases and the surface energy increases. In this regard, in colloidal systems, the interphase surface decreases and the particle distribution density increases, which can lead to instability of the structure, i.e. to a decrease in the degree of dispersion due to the combination of particles. Suspensions, emulsions, foams, aerosols and powders are microheterogeneous systems that represent a wide class of different food products. Suspensions contain a solid dispersed phase in a liquid dispersion medium. A typical slurry process is, for example, a starch making process.

Modeling the dynamic behavior of various food materials, taking into account their rheological characteristics, allows you to choose the optimal processing technology and develop rational designs when designing new processing equipment.

Important rheological phenomena for food technologies include: relaxation and creep phenomena during deformation of various food materials; increase or decrease in viscosity depending on the rate of deformation of the product; the transition of substances from a solid to a fluid state under mechanical action and re-solidification; aftereffect during deformation of substances; dependence of rheological properties on electromagnetic fields; extensibility of high-molecular-weight protein compounds upon deformation.

In the food industry, in modern technologies for the production of suspensions, liquid media and fillers, they are used to obtain a stable consistency, which can be used for the production of pancake tape on existing equipment - Shpakov brazier or pancake, depending on the capacity of the enterprise. The basis of this structure should be conditional liquid media: water, normalized whole milk, reconstituted milk, water-milk mixture. The consistency, structural and mechanical properties and nutritional value of the suspension depend on the liquid portion of the composition used. An important factor is the price of products: a cheap product or a tasty one with increased nutritional value. In order to increase the strength characteristics of the suspension, structure-forming agents and thickeners are currently introduced into the system, which make it possible to obtain products with a stable structure during baking.

Currently, one of the promising directions for increasing the stability of the suspension is the use of stabilizers, emulsifiers and thickeners (emulsifiers E-400 - E-481 and stabilizers for emulsions and suspensions). Emulsifiers contribute to the creation of a uniform consistency of food products, both liquid (preventing the deposition of suspended particles) and solid (which were in a liquid state during the manufacturing process) (Stabilizers, emulsifiers, thickeners, 2021). By entering into chemical reactions with other ingredients, thickeners transform the texture of the dish, making it denser and more uniform. At the same time, substances in this category do not have a pronounced odor and do not have any effect on the taste of the product to which they are added. The use of stabilizers also helps to keep the desserts 
fluffy, preventing the whipped mass from settling. In addition, these additives prevent moisture from excreting in foods, prolonging their freshness.

However, the structure and quality of the suspension directly depends on the quality of the liquid (Thickeners and stabilizers in confectionery production, 2021). Analysis of the state of the problem of water treatment at food industry enterprises according to the available published data showed that a significant part of these enterprises use water from a centralized drinking water supply system. The softening process is mainly carried out by ion exchange. In the case of formation during regeneration of wash water with a concentration of strong $\mathrm{HCl}$, an amount of $2 \%$ is discharged into the sewerage system (Borisov et al., 2014; Golubeva, 2005; Korchagin, 1999). Another method of processing can be proposed - magnetic. This method has no reagents, is more environmentally friendly, devices for its implementation are also more economical compared to traditional water treatment. If the enterprises use underground mines, then the method of cleaning from iron - aeration - is used everywhere (Mazur, 2002; Meledina, 2003; Somin et al., 2015). This is an old and complex technology. In modern conditions, catalytic sorbents are used for these purposes. Therefore, it is desirable to replace aeration with catalytic oxidation. The most likely in this case is the use of Vaiykeg filtration material, which is produced in Russia. There is also adsorption of water on active carbons. A feature of this technology is the complete removal of organic compounds. They are also removed when preparing drinking water for chlorination. Ozonation is a modern method in the production of alcoholic and non-alcoholic beverages. A promising technical solution for water purification from underground sources can be considered water treatment at distilleries: "Kristall" (Kaluga); enterprises of the brewing industry: JSC "Barnaul Brewery", "Stavropol Brewery" (Bannikov, 2004; Jarret \& Ducause, 1998; Krasnova, 2018; Krasnova et al., 2011; Tenju \& Genca, 1988; Ryabchikov, 2013). At the same time, water purification at distilleries and distilleries is complex and requires further development and improvement.

\section{Purpose of the Study}

Currently, one of the promising directions for increasing the set of service properties of these is the introduction of a dispersed powder of biologically active additives into the plant. But the mechanism of interaction of these additives with rice flour, made in the form of a suspension, as well as the technological laws of such a modification, have not been deeply studied. However, this modification with biologically active additives is promising from several points of view. The article presents a study of the influence of various additives of pre-processed tomato powder, parsley, dill, apple, carrot, soybeans and Jerusalem artichoke on the structure of fresh batter.

\section{Research Methods}

As a material for the study, 7 samples of frozen dough with various additives were used: sample 1 - with Jerusalem artichoke - 1.4\%; sample 2-apple-carrot, 1.0\%; sample 3-apple-carrot, 1.5\%; sample 4apple-carrot, 2.0\%; sample 5-tomato, paprika, dill, 1.0\%; test 6-tomato, paprika, dill, 2.0\%; test 7-tomato, paprika, dill, $3.0 \%$. Before starting the analysis, all samples were thawed at room temperature $23{ }^{\circ} \mathrm{C}$ without access to light for 2 hours. Microscopy was performed at $22^{\circ} \mathrm{C}$. One drop of the sample was 
taken and preparations were made with the test and examined under an Olympus BX53 light microscope (Olympus Corporation, Japan) with the eyepiece prefix Carl Zeiss ERc 5s (Zeiss, Germany) and the ZEN lite software (Zeiss, Germany). Microscopy at 50oC. A drop of dough was placed on a beaker and heated in a thermostat at $50^{\circ} \mathrm{C}$ for 10 minutes. The samples were examined under a Micromed MC-5-ZOOM LED microscope. Microscopy. A drop of dough was placed on a beaker and heated in an oven at $100^{\circ} \mathrm{C}$ for 5 minutes. The finished samples were examined under an Olympus BX53 light microscope (Olympus Corporation, Japan) with a Carl Zeiss ERc 5s eye attachment (Zeiss, Germany) and the ZEN lite software (Zeiss, Germany).

\section{Findings}

Based on theoretical assumptions, the main consequence of the introduction of nanopowder of tomato, parsley, dill, apple, carrot, soybeans and Jerusalem artichoke on the structure of fresh batter should be the clarification of the macro- and microstructure, since the powder particles should serve as the nuclei of new grains. Figures 1-3 show photographs of the microstructure of samples of liquid unleavened dough, both modified rice flour from whole grains, dispersed powder of herbal dietary supplements, and without the addition of modifiers. The phase composition of the suspension under study, shown in the photographs of the microstructure, is a solution of rice starch and protein, the inclusion of vegetable and fruit powder, as well as soy protein with the formation of a colloid. Whole milk $2.5 \%$ fat served as a liquid medium in all samples.

At the same time, the addition of such modifiers as herbal additives has little effect on the morphology of a batter consisting of a conditioned aqueous medium and fillers.

To compare the effect of the type of liquid on the suspension, samples of a suspension prepared from rice flour and water at a flour concentration of 1,2, and 3\% were studied in parallel.

Figure 1 shows the microstructures of liquid test suspensions with various concentrations of pretreated powder from uncolored vegetable raw materials with milk (a, b, c) and a suspension of rice water and flour (e, f, g - flour concentration 1, 2 and 3\%. ).

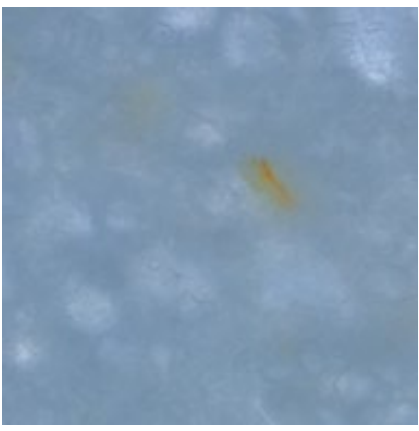

a

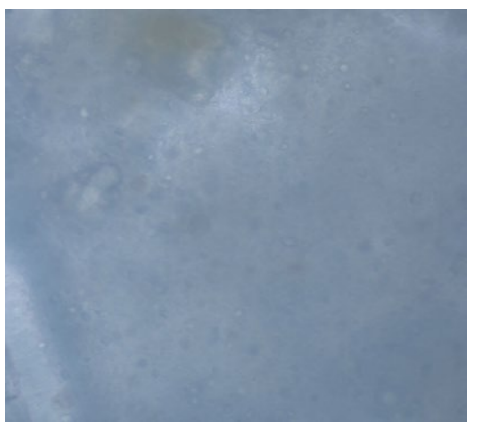

B

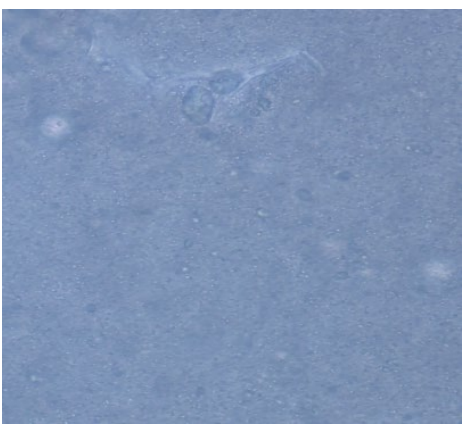

$\mathrm{c}$ 


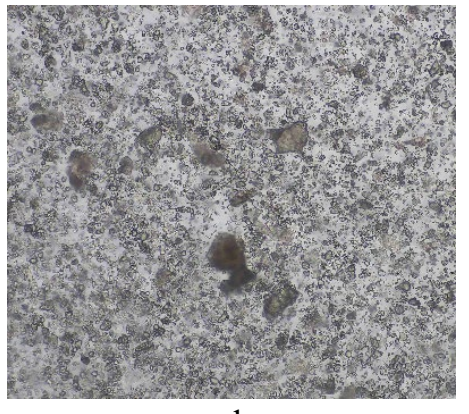

d

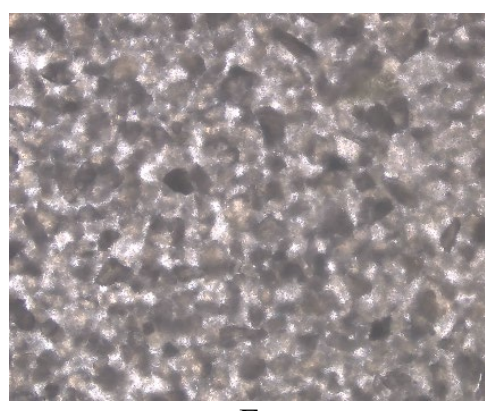

E

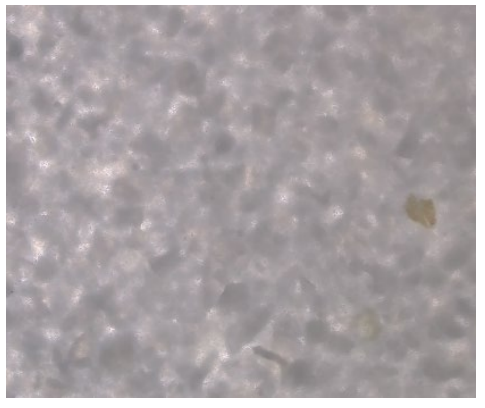

f

Figure 1. . Microstructure of liquid test suspensions with various concentrations of pre-processed powder from uncolored vegetable raw materials with milk: a - with Jerusalem artichoke $1.4 \%$; sample b-apple - carrots, $1.0 \%$; sample c - apple - carrot, $1.5 \%$. Samples e, f, g with water at a concentration of 1,2 , and $3 \%$. Magnification x 10

It was found that the suspension, consisting of rice flour and water, has its own peculiarity: rice flour contains more starch than protein and the pictures show more dense structures with swollen starch grains. At a temperature of $20^{\circ} \mathrm{C}$, starch grains retain their shape well.

The introduction of milk into the suspension leads to an increase in protein in the mixture and the structure becomes denser (Fig. 1: a, b, c). Herbal powders lightly stain micro-slices.

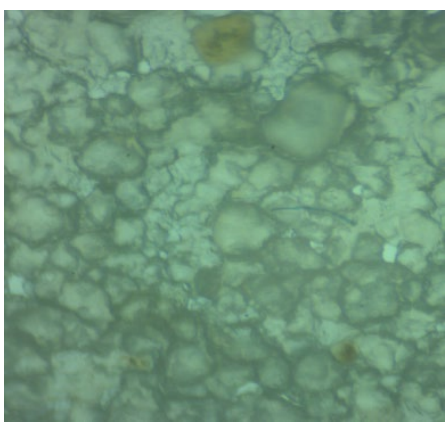

a

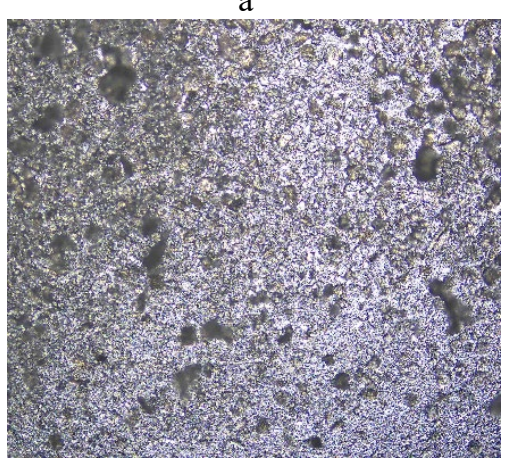

d

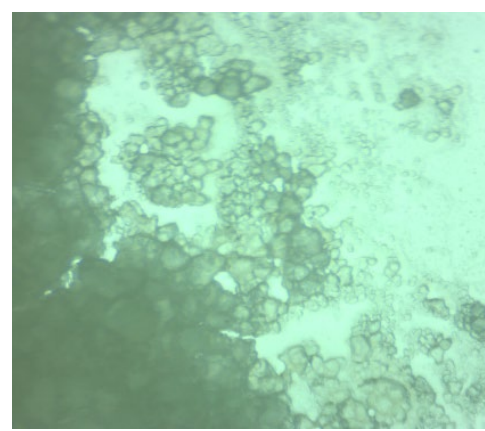

b

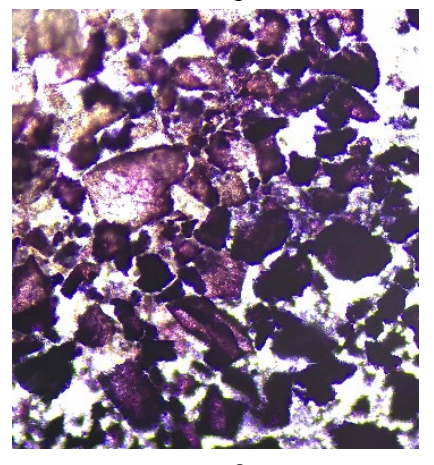

e

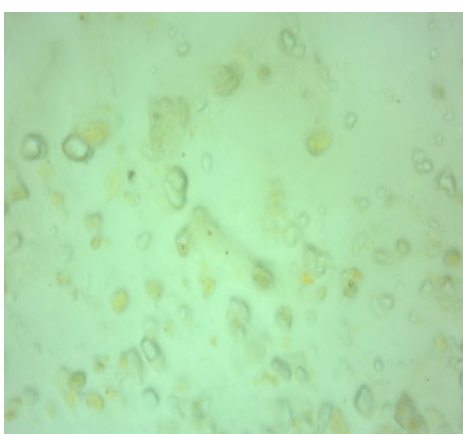

c

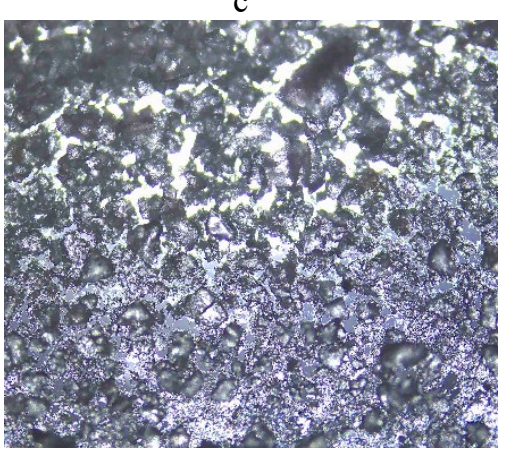

f

Figure 2. Microstructure of a slurry suspension heated in a thermostat at $50^{\circ} \mathrm{C}$ for 10 minutes with different concentrations of pretreated vegetable powder, colored with iodine: sample a Jerusalem artichoke, 1.4\%; tomato, sample b -apple, carrot, $2.0 \%$ and sample c -tomato, paprika, dill. 3.0\%. Samples d, e, g with water at a concentration of 1, 2, and 3\%. Magnification $\mathrm{x} 40$ 


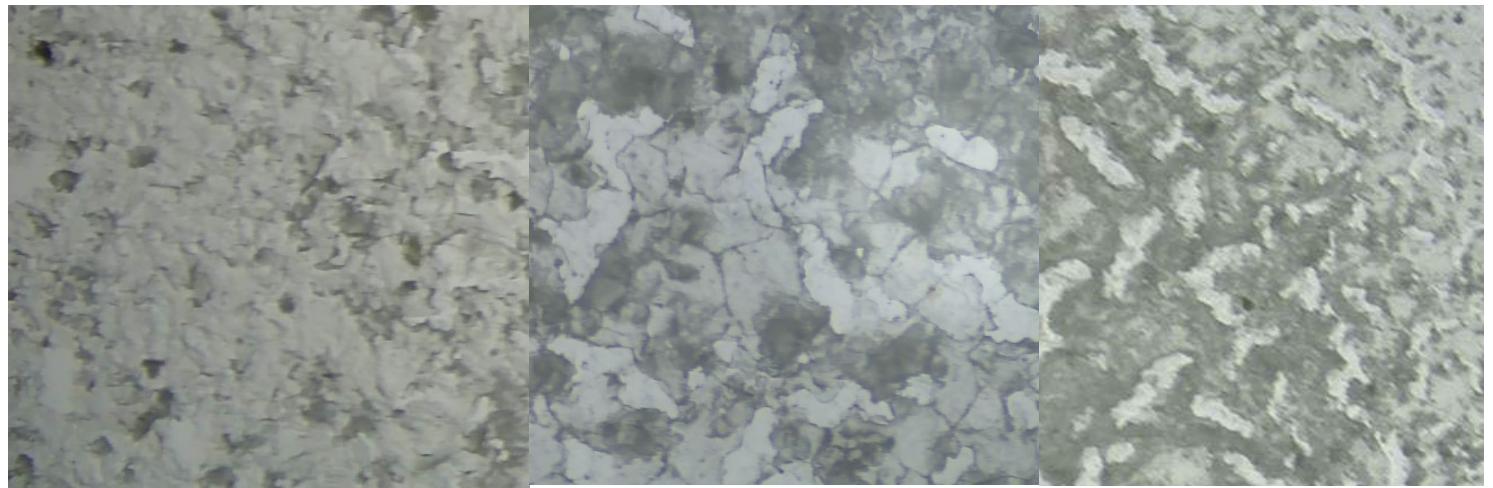

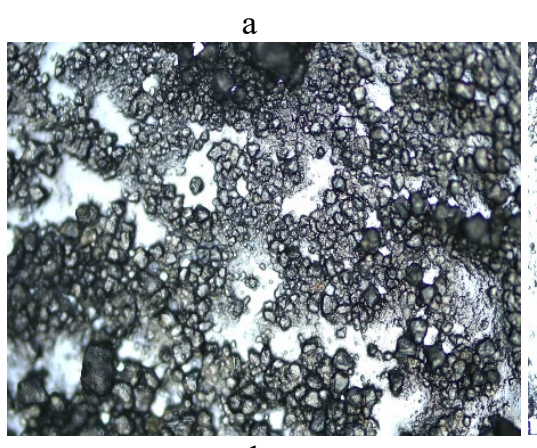

d b

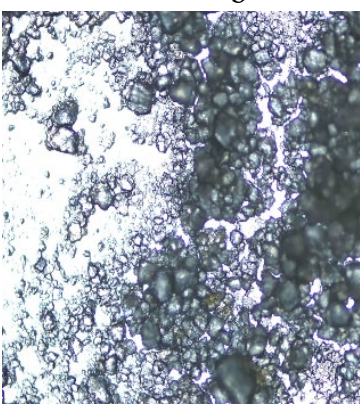

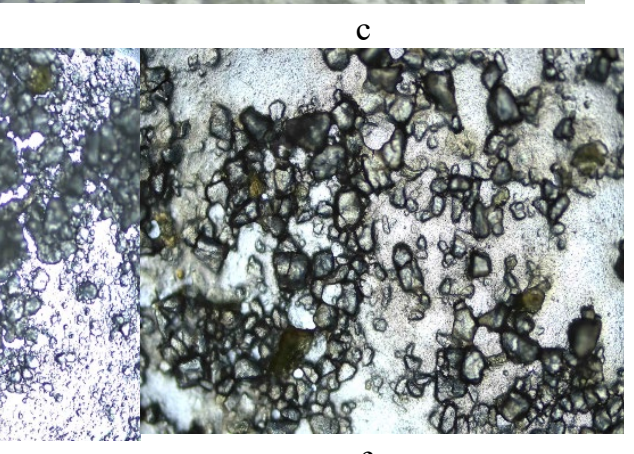

f

Figure 3. Microstructure of a slurry suspension heated in a thermostat at $100^{\circ} \mathrm{C}$ for 5 minutes with different concentrations of pretreated vegetable powder, colored with iodine: sample a Jerusalem artichoke, 1.4\%; tomato, sample b -apple, carrot, $2.0 \%$ and sample c -tomato, paprika, dill. 3.0\%. Samples d, e, g with water at a concentration of 1, 2, and 3\%.

Magnification x40

As can be seen from Figures 1-3, as a result of modification of crystallization conditions, various morphologies of inclusions of plant powders are formed in rice suspension prepared in liquid media. In addition, it is known from previous studies (Kolomnikova et al., 2015; Vasilenko et al., 2018; Vasyukova et al., 2019; Vasyukova et al., 2020) that the cooling rate significantly affects both the shape and the number of clots during formation. The higher the cooling rate, the less protein-starch gel is formed.

Figures 1-3 show that the addition of a small amount of plant powder $(1.0 \ldots 3.0 \%)$ makes it possible to modify the microstructure of the resulting suspensions. This modification includes the refining of rice starch grains, stabilization of the non-splitting structure with reduced sizes of matrix inclusions of solid particles of plant biologically active additives, as well as the formation of spherical inclusions of rice starch pasteurized at 50 and $100 \mathrm{oC}$. At the same time, the addition of such modifiers as herbal additives has little effect on the morphology of a batter consisting of a conditioned aqueous medium and fillers.

Heating of the samples resulted in partial $\left(\right.$ at $50^{\circ} \mathrm{C}$ ) and complete (at $100^{\circ} \mathrm{C}$ ) gelatinization of rice starch and denaturation of orysenin. A qualitative analysis of the structure of the samples of the liquid suspension of the test showed that we could not find a significant difference in the number of clots at different concentrations of the modifier. With the help of microscopic studies, it was found that the change in the content of clots is not as significant as it was under the influence of low temperatures. The maximum difference in clot reduction with the addition of $3.0 \%$ tomato, paprika, dill was found in the 
case of a sample that contained $27.0 \%$ of the modifier. The samples with the addition of the modifier $39.0 \%$ and $48.0 \%$ had a highly branching clot morphology.

After the conducted studies of the suspension of the liquid dough, it was shown that the structures underwent changes, which allows us to conclude that the modification of dispersed powders of plant products does not significantly affect its structure. This is especially true for low concentrations of the modifier (up to $27.0 \%$ ). With the introduction of a small amount of powder from plant materials, the distance between pasteurized grains of rice starch and oryzenin decreases. This means that a significant part of the powder particles of herbal dietary supplements are effective centers of gelation. With an increase in the content of nanopowder, the structure begins to coarse relative to that obtained with the introduction of low concentrations of the powder. The addition of a large amount of the modifier (up to $48.0 \%$ ) leads to its coagulation and a decrease in the effect on the structure. Pasteurized and colored starch in all samples was characterized by the formation of a colloid. At the same time, the addition of such modifiers as herbal additives has little effect on the morphology of a batter consisting of a conditioned aqueous medium and fillers.

An increase in temperature to 50 and $100^{\circ} \mathrm{C}$ promotes an increase in rice starch gelatinization and the formation of a liquid starch paste in samples with water and rice flour with a concentration of 1,2 , and 3\% (Fig. 2: 1, m, n - at $50^{\circ}$ C); (Fig. 3: o, n, p - at 100oC). An increase in temperature leads to the formation of clumps of glued starch with a dense branched structure.

\section{Conclusion}

The rheological properties of concentrated suspensions of polar and non-polar particles have been investigated. The main attention was paid to the influence of heterogeneous aggregates on the rheological properties of these systems. A microscopic model of shear hardening of highly concentrated suspensions based on the mechanism of dry contact between particles has been developed. The developed model made it possible for the first time to consistently describe experiments where suspension hardening by the introduction of plant powders was observed. The features of the rheological properties of concentrated suspensions have been investigated.

\section{References}

Bannikov, B. (2004). Electromagnetic water treatment: the device "Termite". Ecology of production, 4, 25-32.

Borisov, B. A., Egorova, E. Y., \& Zainullin, R. A. (2014). Water treatment in the production of food and beverages. Professiya.

Golubeva, L. V. (2005). Directory of dairy technologist. GJORD.

Jarret, M., \& Ducause, C. (1988). Influence du climat et des treitements de potabilisation sur Toxidabilite d'une eau en cours d'affmage [Influence of climate and drinking water treatment on the Toxicity of a water in the process of starving Rev. Sci. Water] Rev. Sci. Eau, 1(4), 355-383. https://doi.org/10.7202/705017ar

Kolomnikova, Y. P., Derkanosova, A. A., Manukovskaya, M. V., \& Litvinova, E. V. (2015). Effect of non-traditional vegetable raw materials on the properties and biotechnological structure pastry. Proceedings of the Voronezh State University of Engineering Technologies, 3, 157-160. (In Russ.) https://doi.org/10.20914/2310-1202-2015-3-157-160 
Korchagin, V. I. (1999). Dependence of properties of semi-finished products and finished products on the chemical composition of water. Bakery of Russia, 6, 22-23.

Krasnova, T. A. (2018). Water treatment in the foodindustry. Equipment and technology of food production, 48(1), 15-30. https://doi.org/10.21603/2074-9414-2018-1-15-30

Krasnova, T. A., Yustratov, V. P., \& Poznyakovsky, V. M. (2011). Examination of drinking water. Quality and safety. DeLi print.

Mazur, P. Y. (2002). Yansheva IN Voda V prigotovlenii khleba [Water in Bread Production]. Khlebopecheniye Rossii, 30-32.

Meledina, T. B. (2003). Raw materials and auxiliary materials in brewing. Professiya.

Ryabchikov, B. E. (2013). Modern water treatment. DeLi print.

Somin, B. A., Kurtukova, L. V., \& Komarova, L. F. (2015). Groundwater softening using a new sorbent based on bentonite clays. Ecology and industry of Russia, 1, 30-33.

Stabilizers, emulsifiers, thickeners. (2021). https://www.babyblog.ru/user/Krysya /402077

Thickeners and stabilizers in confectionery production. (2021). https://www.zeelandia.ru/blog/naturalnyestabilizatory-zagustiteli-v-konditerskom-proizvodstve

Tenju, P., \& Genca, K. (1988). Zur Nutzung von Aktivkohle zur Trinkwasseraufbereitung in del VB Bulgarien Wasserwirt [For the use of activated carbon for drinking water treatment in the VB Bulgaria water management]. Wassertechn, 38(2), 33-34.

Vasilenko, V. N., Frolova, L. N., Derkanosova, A. A., Mikhailova, N. A., Shhepkina, A. A., \& Davydov, A. M. (2018). Software of the extrusion process abnormally viscous fluids methods of experiment planning. Proceedings of the Voronezh State University of Engineering Technologies, 80(3), 3742. (In Russ.) https://doi.org/10.20914/2310-1202-2018-3-37-42

Vasyukova, A. T., Alekseev, A. A., Slavyansky, A., \& Moshkin, A. B. (2019). The use of natural plant supplements in flour culinary products. III International Scientific and Practical Conference "Science and Education in the Modern World: Challenges of the Twentieth Century", 289-293.

Vasyukova, A. T., Ganina, V. I., \& Egorova, S. V. (2020). The dietary supplement: Composition, control and functional properties. Journal of Advanced Research in Dynamical and Control Systems, 12(4), 903-906. https://doi.org/10.5373/JARDCS/V12SP4/20201560 